\title{
Imagerie normale du confluent vésiculo-deferentiel
}

\author{
A. Dana, B. Martin, C. Rov \\ Institut de Radiologie, 31 Avenue Hoche, 75008 Paris
}

\section{RESUME}

Les techniques de visualisation directe de la voie séminale se sont multipliées.

A côté de l'opacification directe de la voie séminale, technique anciennement connue, se sont développées successivement l'échographie et l'imagerie par résonance magnétique ; ces deux techniques utilisent la voie endorectale pour la sonde dans le premier cas et pour l'antenne dans le deuxième.

Ces deux techniques présentent l'avantage d'une innocuité parfaite.

Mots clés : Déférentographie, Echographie endo-rectale, Imagerie par résonance magnétique, Antenne endo-rectale

\section{INTRODUCTION}

L'imagerie de la voie séminale a été révolutionnée par l'apparition successive de l'échographie et de l'IRM avec antenne endorectale.

Après un rappel des données de la déférentographie, nous étudierons les aspects normaux que démontrent ces deux techniques.

\section{LA DEFERENTOGRAPHIE : ASPECTS NORMAUX}

L'opacification globale du canal déférent est obtenue par la déférentographie dont la technique et les résultats sont parfaitement détaillés dans l'ouvrage de Boreau [1].
Les clichés sont réalisés de face avec une obliquité variable du rayon directeur.

A l'état normal, le canal déférent est visible sur tout son trajet selon un fin conduit de calibre régulier.

L'opacification permet également la visualisation du canal éjaculateur et de la vésicule séminale.

Cette technique autorise l'opacification de l'anse épididymo-déférentielle et de l'épididyme.

\section{ASPECTS ECHOGRAPHIQUES DU CONFLUENT VESICULO-DEFEREN- TIEL $[2,4,5]$}

Le confluent vésiculo-déférentiel représente la réunion de la vésicule séminale et $\mathrm{du}$ canal déférent. Il se prolonge par le canal éjaculateur.

D'un point de vue technique, l'échographie doit être réalisée par voie endorectale. L'examen par voie sus-pubienne, réalisé avec une vessie pleine, ne permet pas une étude satisfaisante des vésicules séminales et du confluent vésiculo-déférentiel.

L'examen échographique est ainsi réalisé avec une sonde endorectale, multiplans ; la fréquence est de l'ordre de $7,5 \mathrm{MHz}$.

Les constructeurs s'orientent actuellement vers des sondes multifréquences qui peuvent varier de 6 à $10 \mathrm{MHz}$.

\section{Résultats}

Les vésicules séminales sont surtout 
visibles sur les coupes transversales: elles sont anéchogènes ou hypo-échogènes. Elles sont parfois asymétriques et présentent volontiers une structure aréolaire ; elles sont situées en arrière de la vessie, immédiatement au-dessus de la prostate (Fig.1). Les ampoules déférentielles sont situées en avant de la partie interne des vésicules séminales.

Le confluent vésiculo-déférentiel est représenté par un triangle hypo-échogène qui pénètre la zone centrale de la prostate, obliquement en bas et en-dedans (Fig.2).

Ce confluent se prolonge par le canal éjaculateur, rarement visible sous la forme d'un fin liseré hypo-échogène.

Sur les coupes transversales obliques, les deux confluents vésiculo-déférentiels sont visibles selon une zone plus ou moins triangulaire médiane à base antérieure, immédiatement en avant du tissu prostatique.

Les canaux éjaculateurs, rarement visibles séparément, peuvent globalement être représentés par une zone nodulaire, arrondie, hypo-échogène, médiane, en arrière de l'urètre et au-dessus du veru montanum où ils s'abouchent.

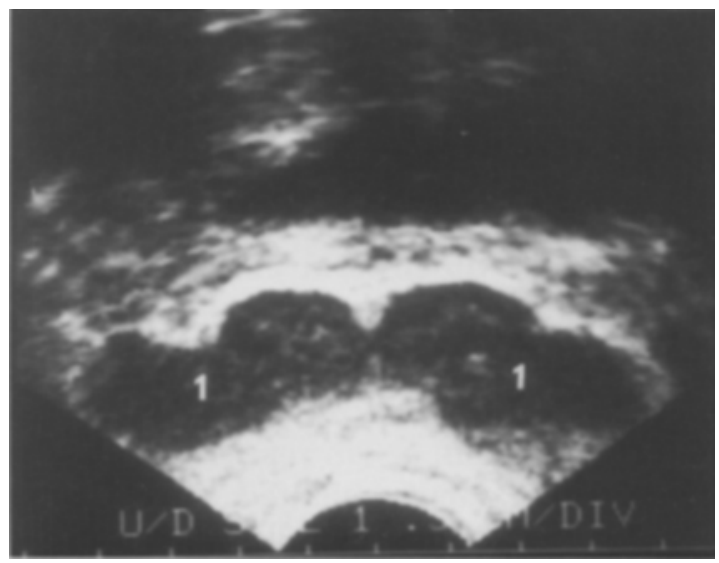

Figure 1 : echographie endo-rectale : vésicules séminales symétriques, hypoéchogènes.

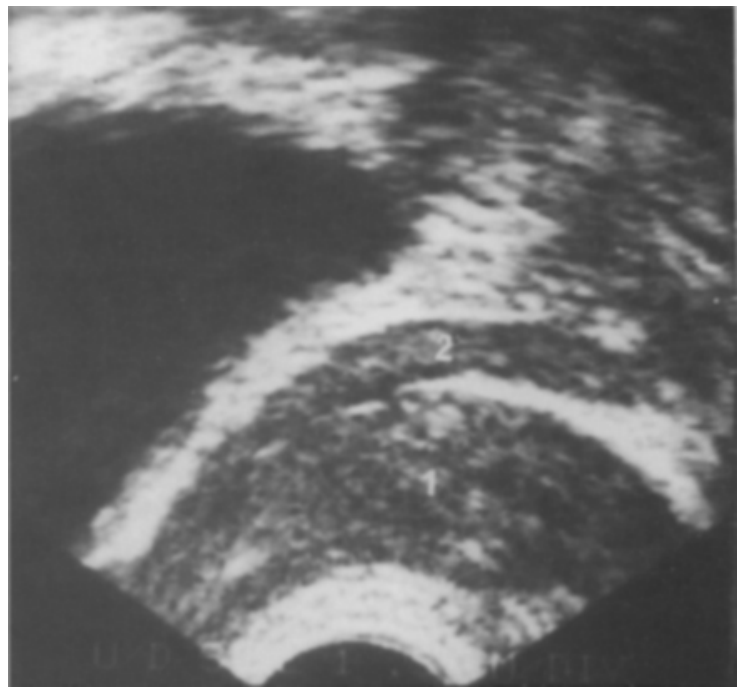

Figure 2 : echographie endorectale : bonne visibilité du confluent vésiculodéférentiel.

\section{3) ASPECTS IRM DU CONFLUENT VESICULO-DEFERENTIEL [3]}

\section{Technique}

L'examen est pratiqué au moyen d'une antenne endo-rectale conçue sur le modèle désormais classique (Medrad, Pittsburgh), antenne de surface placée à l'intérieur d'un ballonnet, montée sur un support rigide, gradué en centimètres, permettant une évaluation précise de la mise en place de l'antenne par rapport à la marge anale.

L'injection de Glucagon, en réduisant la motilité rectosigmoïdienne et les artefacts qui en résultent, améliore significativement la qualité des images.

La mise en place de l'antenne est toujours précédée par un toucher rectal, avec un doigtier enduit de gel de Xylocaïne . L'antenne est ensuite introduite, ballonnet dégonflé. Le ballonnet est ensuite progressivement gonflé avec $80 \mathrm{cc}$ d'air.

Deux séquences de coupes transversales sont successivement obtenues, en technique Spin Echo. La première séquence est pon- 
dérée en T2, la seconde est pondérée en T1, après injection de Gadolinium. Pour cette séquence, la suppression de graisse est utile car elle améliore la qualité des images.

Enfin, une série de coupes frontales, dans l'axe du confluent vésiculo-déférentiel , en pondération $\mathrm{T} 1$, permet l'analyse du pied des vésicules séminales.

\section{Résultats}

Les vésicules séminales sont de morphologie variable, mais en règle, de signal similaire.

En T1, elles sont homogènes de signal intermédiaire.

Après injection de Gadolinium, l'architecture interne des vésicules séminales est bien mise en évidence, avec un aspect aréolaire : les logettes en hyposignal étant entourées par des septa "blancs" en hypersignal (Fig.3).

En T2, les vésicules séminales sont en hypersignal, avec une excellente visibilité des septa qui sont en hyposignal (Fig.4).

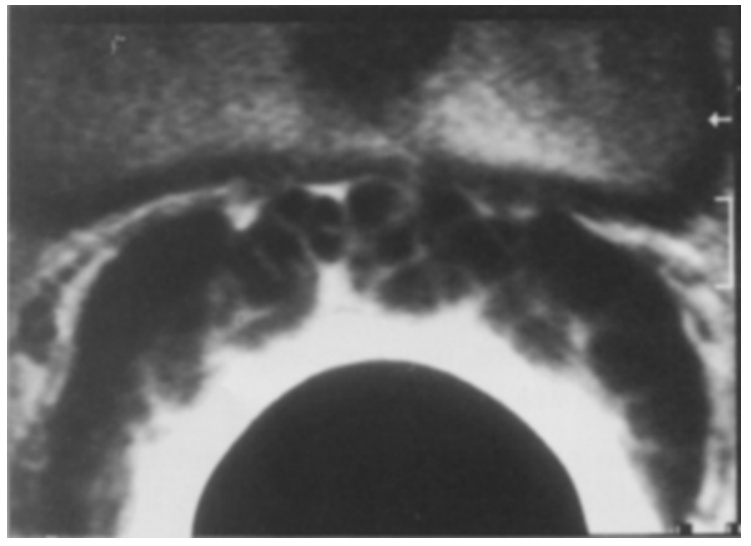

Figure 3 : irm endorectale : séquences pondérées en t1. les vésicules séminales sont représentées par des logettes hypo-intenses, séparées par des septa hyper-intenses.

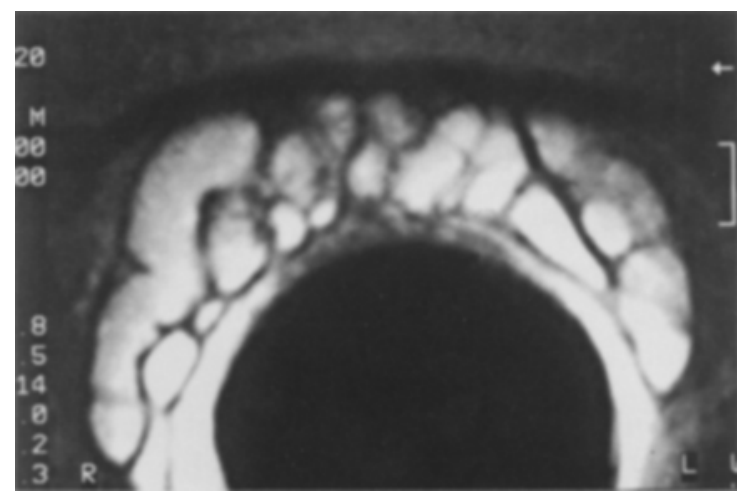

Figure 4 : irm endorectale : séquences pondérées en 12 . logettes hyper-intenses, séparées par des septa hypo-intenses.

Parfois, les vésicules séminales restent en hyposignal du fait d'un défaut de liquide circulant dans des vésicules scléro-atrophiques.

Les ampoules déférentielles situées à la partie interne des vésicules séminales sont volontiers représentées par une zone plus ou moins arrondie en hyposignal, du fait de la présence d'une faible quantité de liquide à leur niveau.

Le confluent vésiculo-déférentiel est par ailleurs bien analysé sur les coupes frontales avec une meilleure appréciation de l'hypersignal relatif en T2.

\section{CONCLUSION}

Les techniques endorectales aussi bien en échographie qu'en IRM permettent une analyse fine de la voie séminale, et notamment du confluent vésiculo-déferentiel.

Ces techniques sont dépourvues de tout caractère invasif, ce qui représente un progrès réel par rapport aux techniques d'opacification directe. 


\section{REFERENCES}

1. BOREAU J. Les images des voies séminales. S. KARGER edit. Paris, 1974

2. DANA A. Atlas d'échographie de la prostateMasson Edit.1992

3. DANA A. Imagerie de la prostate Masson Edit. 1994

4. MARTIN. B Atlas d'échographie scrotale. Springer-Verlag Edit. 1992

5. RESNICK M.I. Echographie prostatique. Pradel Edit. 1991

\section{ABSTRACT \\ NORMAL IMAGING OF JUNCTION OF THE VAS DEFERENS AND SEMINAL VESICLE}
A. Dana, B.Martin, C.RoY

Imaging modalities for direct visualization of the seminal tract have changed during the last ten years.

The opacification of the seminal tract by contrast agents, which is an invasive procedure is now less performed and new non invasive imaging modalities are available : ultrasounds using a high frequency $10 \mathrm{MHz}$ endorectal probe and Magnetic Resonance Imaging using an endorectal coil.

Key Words : deferentography, endorectal ultra-sonography, magnetic resonance imaging, endorectal probe 